\title{
Effects of Electrical Stimulation and Electrocoagulation in Cortex and Thalamus on Delayed Response in Monkeys
}

\author{
M. E. OLdS \\ Brain Research Laboratory, Department of Psychology, University of Michigan, \\ Ann Arbor, Michigan \\ Received November 15,1965
}

\begin{abstract}
Eighteen brain points in prefrontal and thalamic areas of each of three adult male macaques were tested for the effects of electrical stimulation and coagulation during the delayed response test. During the delay period, stimulation of prefrontal areas and dorsumedial thalamus caused severe impairment in two subjects. Stimulation caused less impairment during the baiting period than during the delay period; continuous stimulation throughout the test caused less impairment than intermittent stimulation; intermittent stimulation throughout the test was the most effective mode of stimulation. Distraction tests produced the same standard of performance achieved during control tests; peripheral shocks produced a slightly impaired performance. Even aversive peripheral stimuli did not produce as many errors as did central stimuli which produced no behavioral effects. Prefrontal lesions had more disruptive and longer-lasting effects than did thalamic lesions, but the order in which the lesions were made had no effect on performance. The results substantiate previous conclusions that electrical stimulation of points along the banks of the principal sulcus can impair the delayed response performance of overtrained subjects, but, on the other hand, stimulation of dorsomedial nucleus of thalamus has the same effect.
\end{abstract}

\section{Introduction}

Bilateral resection of prefrontal areas in monkey brains results in deterioration of the animals' ability to perform the delaycd response test. However, studies involving electrical stimulation of these areas have yielded less definite results. Weiskrantz, Mihailovic and Gross (6) showed that intermittent stimulation throughout the delayed alternation test caused severe but reversible deficits in well-trained subjects. Stamm (4) on

1 This study was supported by a grant from the U. S. Public Health Service to The University of Michigan to support the research of Dr. James Olds and by grants from Hoffmann-La Roche Inc. and Wallace Laboratories to Dr. M. E. Olds. 
the other hand found that stimulation of prefrontal areas produced deficits which were inversely related to learning: Excitation had no effect on overtrained subjects but caused large deficits during learning of the delayed response task. Rosvold and Delgado (3) showed that stimulation and electrocoagulation in the caudate nucleus caused deficits in performance of delayed alternation, a task similar in many respects to delayed response. Studies of Cianci (2) and Briese and Olds (1), also using electrical stimulation, pointed to prefrontal areas and to the dorsomedial nucleus of the thalamus as structures where excitation caused deficits on delayed response tests.

In view of the inconclusiveness of the results obtained by electrical stimulation, the present experiments were carried out to determine whether the results obtained earlier could be substantiated using various stimulation techniques, and whether the prefrontal and thalamic areas were the two ends of a corticosubcortical pathway mediating a delayed response type of behavior.

\section{Methods}

Three adult male macaques ( $M$. cynomolgi) weighing $3-4 \mathrm{~kg}$ served as subjects; a fourth did not survive surgery. The animals, $L, M$ and $N$, were permanently seated in primate chairs modified to include components of the Wisconsin General Test Apparatus. The problem surface had three food wells. A three-choice task with 6-sec delay was chosen to make each trial maximally informative while permitting rapid training and an almost perfect level of responding. The food wells were covered by identical lids which the animal could push separately to expose their contents. Two barriers were suspended by pulleys between the subject and the wells: a transparent one to permit the subject to view the baiting; and an opaque one to hide the wells from view during the retention (i.e., delay) period.

Before any training took place, Formvar-insulated stainless steel electrodes $250 \mu$ in diameter were implanted; only the cut end was uninsulated. Operations were performed under sodium pentabarbital (34 $\mathrm{mg} / \mathrm{kg}$ ). Electrodes impinged on nine points in the dorsomedial nucleus of the thalamus and on nine points along the principal sulcus, on each side of the brain (Fig. 1). Ground electrodes of uninsulated stainless steel wire were implanted in the brain itself. Stimulation was bipolar. During any given test, electrical stimulation was applied simultaneously to all points in the prefrontal areas or to all points in the thalamus. Monopolar 
recordings were taken on an Offner inkwriter from six points at a time, immediately after stimulation of these points, in order to determine whether excitation had caused afterdischarges.

Delayed response training was started 2 weeks after surgery. It consisted of a three-choice test with an intertrial period of $30 \mathrm{sec}$. Small bits of monkey pellets (1-cm cube) were used as the reward. A 22-hour food deprivation schedule was observed with water present at all times. Each

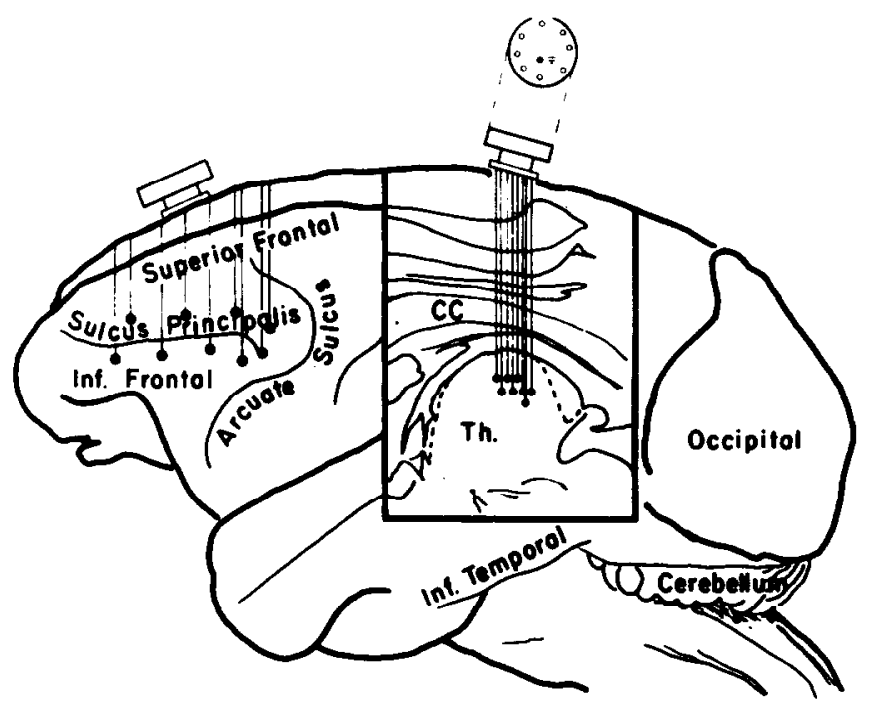

Fig. 1. Sketch showing place-cut of electrode assembly.

monkey was given twenty trials daily. At the end of testing, each was given fifteen pellets to supplement the food obtained during the testing session. This constituted a "control day." Testing was done daily, Monday through Friday. The criterion was met when the subjects' performance on five consecutive daily tests had no more than ten total errors. Subjects required 1000-1500 trials before reaching a level of performancc where the criterion was achieved repeatedly. When stimulation began, the subjects were overtrained.

The experimental design consisted in using each animal as his own control. Electrical stimuli were applied either to frontal areas or to thalamic points, but never to points in both structures simultaneously. Effects of stimulation were studied under four conditions: during baiting; during the delay; throughout the trial with intermittent trains; through- 
out the trial with continuous trains. To minimize the effects of order of stimulation, two of the subjects were tested with initial stimuli applied first to frontal points, and one was tested with initial stimuli applied first to thalamic points. Three control days were alternated with two stimulation days during any given 5-day sequence of tests.

Two special tests were made to compare the effects of central stimulation during the delay period with the effects produced by weak and strong distractions. In one test, the experimenter produced noises by rattling the barriers, etc. In the other test, peripheral electrical shocks were applied via the metal bars of the primate chair. The shocks were varied in intensity to prevent toleration; however, they caused distraction, discomfort and, at times, annoyance with concomitant attempts to escape. ${ }^{2}$ The distraction tests were given after tests inquiring into the effects of stimulating the cortex and the thalamus were completed.

The reinforcement properties of each point excited during the delayed response tests were assessed by the methods of self-stimulation and escape from centrally applied shock. The approach test consisted of a training period during which the animal was brought repeatedly into contact with a lever which caused delivery of a 0.25 -sec, 60 -cycle $/ \mathrm{sec}$ sine wave to one of the points in the prefrontal area or in the thalamus. Subsequent voluntary depression of the lever was used as the measure of positive reinforcement. To measure negative reinforcement, brief trains of electrical stimuli of the same characteristics were applied at the rate of 1 per sec until the animal responded by pressing a lever which caused the series of pulses to be interrupted and postponed for a period of $4 \mathrm{sec}$." These methods have been described in more detail by both Cianci (2) and Briese and Olds (1).

Electrocoagulation studies were started when all stimulation tests were completed and after a control testing period was carried out to re-establish baseline performance levels. Two subjects received their first lesions on all thalamic probes; one subject on the frontal probes. Testing procedures to assess effects of lesions were essentially the same as those made to

2 Application of electrical stimuli via the metal bars was not intended to induce pain. When identical current was applied to the experimenter himself, only a tingling sensation was felt, similar to that when one's foot "goes to sleep."

3 When points in the brain were tested for aversive motivational properties, current was raised progressively to below seizure level; but at no time did the subject move a lever to stop the stimulus, nor make noises suggesting that he experienced even annoyance. 
assess effects of electrical stimulation. If criterion was reached (no more than ten errors on 5 consecutive testing days) larger lesions were made to the same points. If criterion was again reached after a number of tests the subject that had received frontal lesions was given bilateral thalamic ones to all points at once, and the animal which had initially received thalamic lesions was given bilateral multiple frontal lesions. Testing was continued until there was no recovery from the initial deficits caused by electrocoagulation or until death ensued. Brains were embedded in celloidin or frozen, section and stained to assess loci of stimulation and lesion points (Fig. 1).

\section{Results}

During the delay period, stimulation of both the prefrontal areas and the dorsomedial thalamus caused severe impairment in two of the three subjects (Fig. 2). The third subject, $N$, showed almost no impairment. In subjects $M$ and $L$ frontal stimulation was regularly effective at current levels which produced neither behavioral nor abnormal EEG patterns. Sometimes a milder stimulus produced habituation which was overcome by an increase of current. There was also, however, a countertrend of potentiation which resulted in increased impairment or which permitted current reduction without diminishing its effects. The effects of thalamic stimulation during delay were initially as great as those obtained with frontal stimulation. However, there was more adaptation over time to the effects of thalamic stimulation than to the effects of frontal stimulation, except for subject $M$, in which thalamic stimulation and frontal stimulation were equally effective, both showing a similar pattern of effects over time.

The effects of stimulation were recorded from the points stimulated. No signs of afterdischarge appeared at any of the various current levels used. If, on any given test day, stimulation caused seizures, tests for the day were discontinued. During stimulation tests the subjects' speed of performance, behavior and appearance were identical to that observed during the prestimulation tests except that more errors were made.

Scores achieved on control days, alternating with stimulation days, were equal to or even better than scores achieved on prestimulation tests. Thus, the previous day's stimulation had no clear-cut effect on the following day's performance. Repeated stimulation over a number of weeks did not cause the performance to deteriorate (Fig. 3).

In subject $L$ current levels used to stimulate either frontal or thalamic 
points remained at the minimum starting point because higher current caused seizures. Nevertheless, disruption caused by excitation of the prefrontal and thalamic areas during the delay period caused strong im-

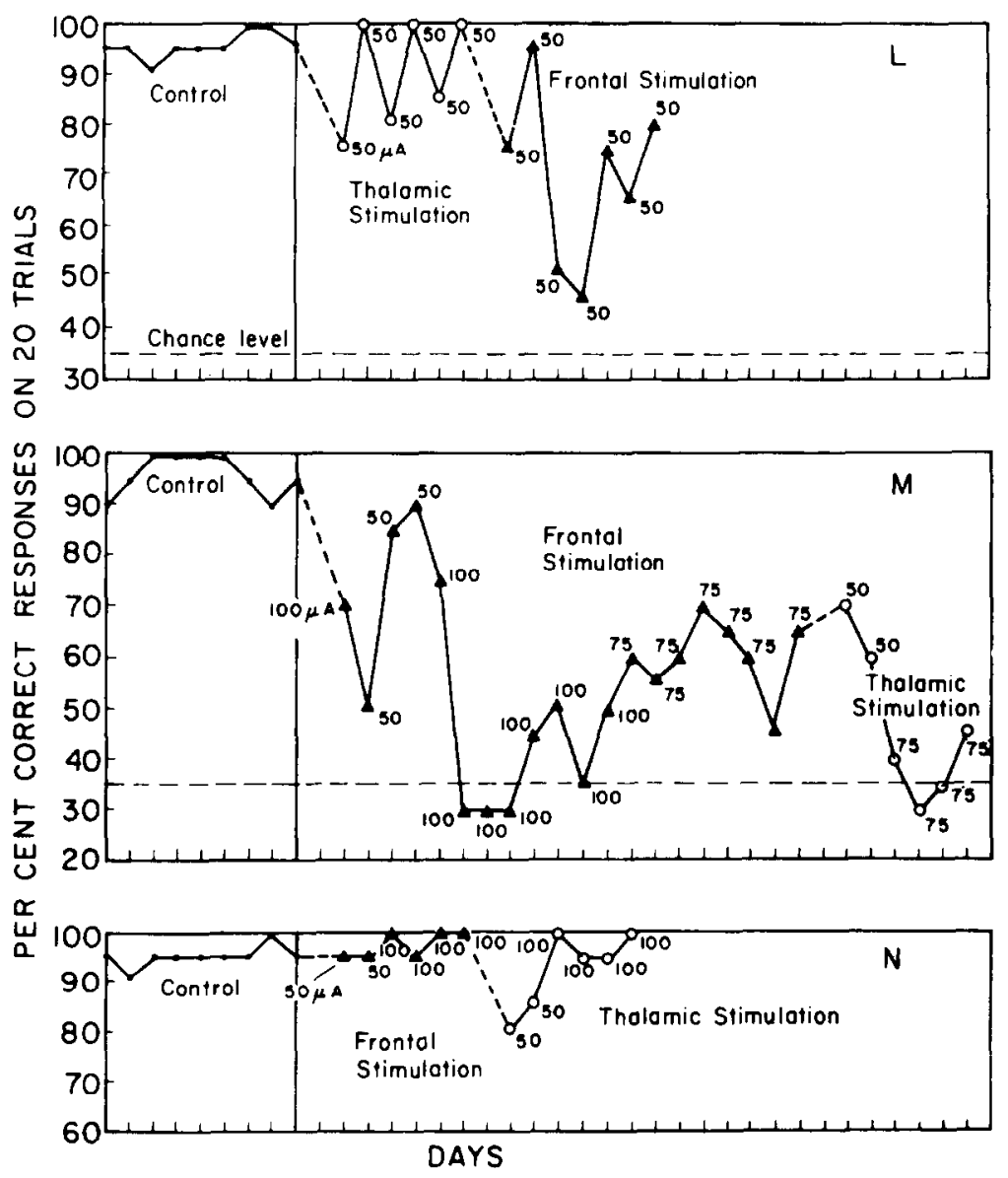

Fig. 2. Effects of electrical stimulation during delay interval, 0.25 sec on, 0.75 sec off; five trains 60 -cycle/sec sine wave.

pairment of $L$ 's performance on the delayed response test even at this low current level.

When stimulation was applied during the baiting period, that is, from the moment the opaque screen was raised and the subject's attention sought, to the moment the opaque screen was lowered, the performance 

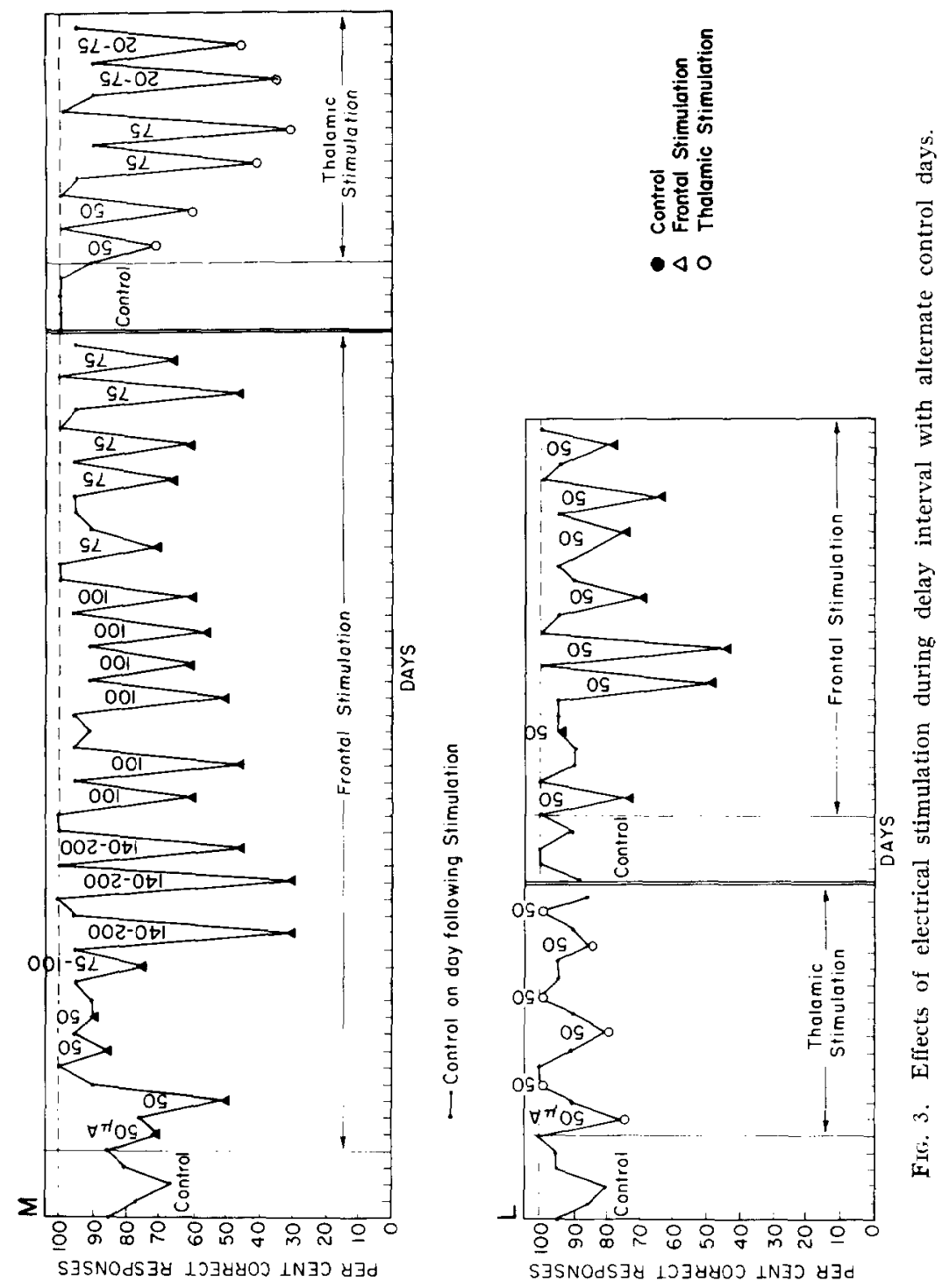
was not impaired as much as when such stimulation was applied during the delay period (Fig. 4).

During the baiting period, thalamic excitation in subject $M$ caused performance to drop to the $60 \%$ correct level, and frontal excitation caused

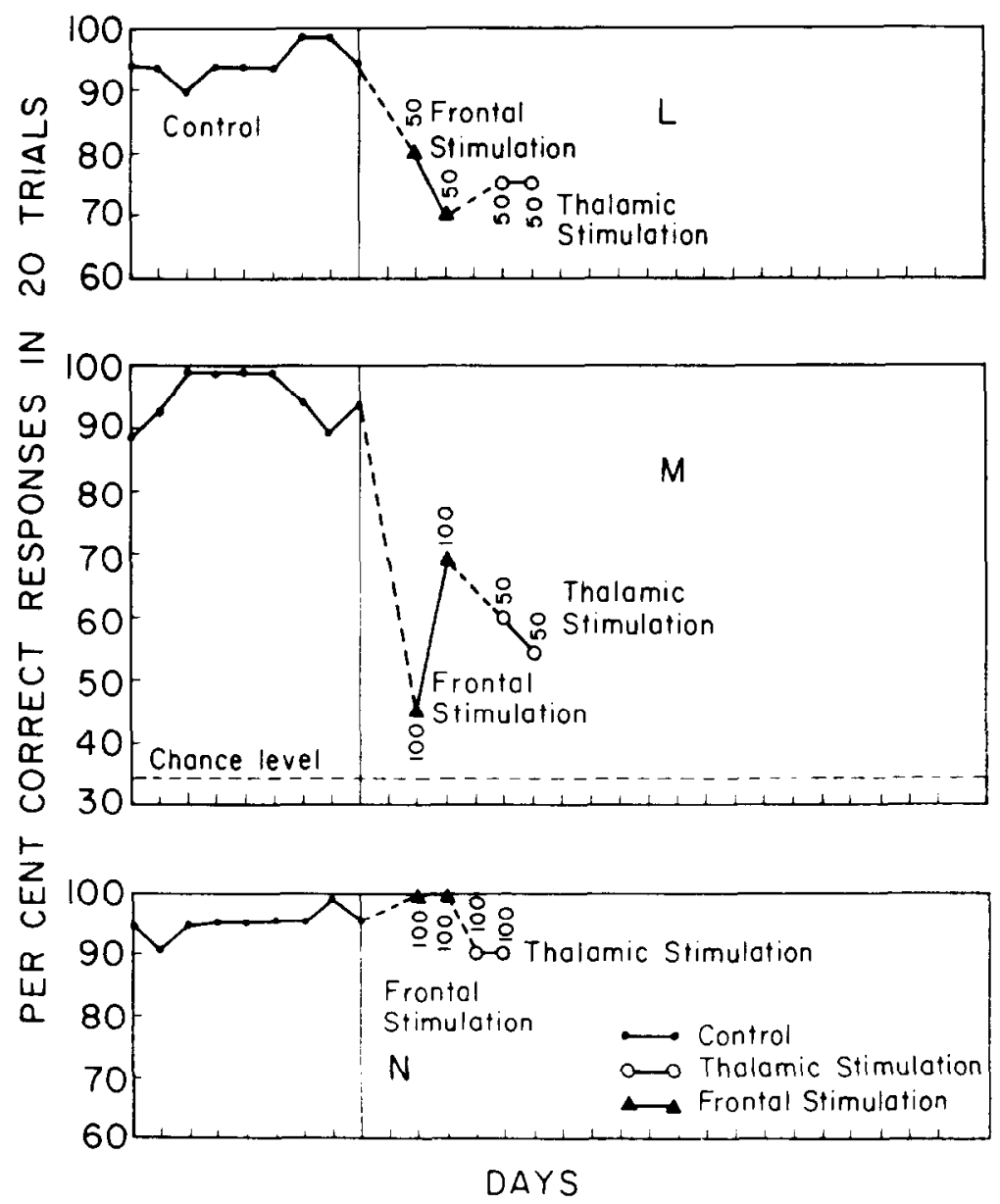

FIG. 4. Effects of stimulation during the baiting interval, $0.25 \mathrm{sec}$ on, $0.75 \mathrm{sec}$ off; five trains $60 \mathrm{cycle} / \mathrm{sec}$ sine wave.

performance to drop to the same level. When a day of control tests was alternated with a day of stimulation tests, no carry-over, or residue, of stimulation was observed on the control day. Subject $N$ again showed 
no difference in effect from application of electrical stimuli either to frontal or to thalamic points.

When intermittent stimulation of 1-sec duration was given once every 4 sec throughout the test, impairment was severe in two of the three

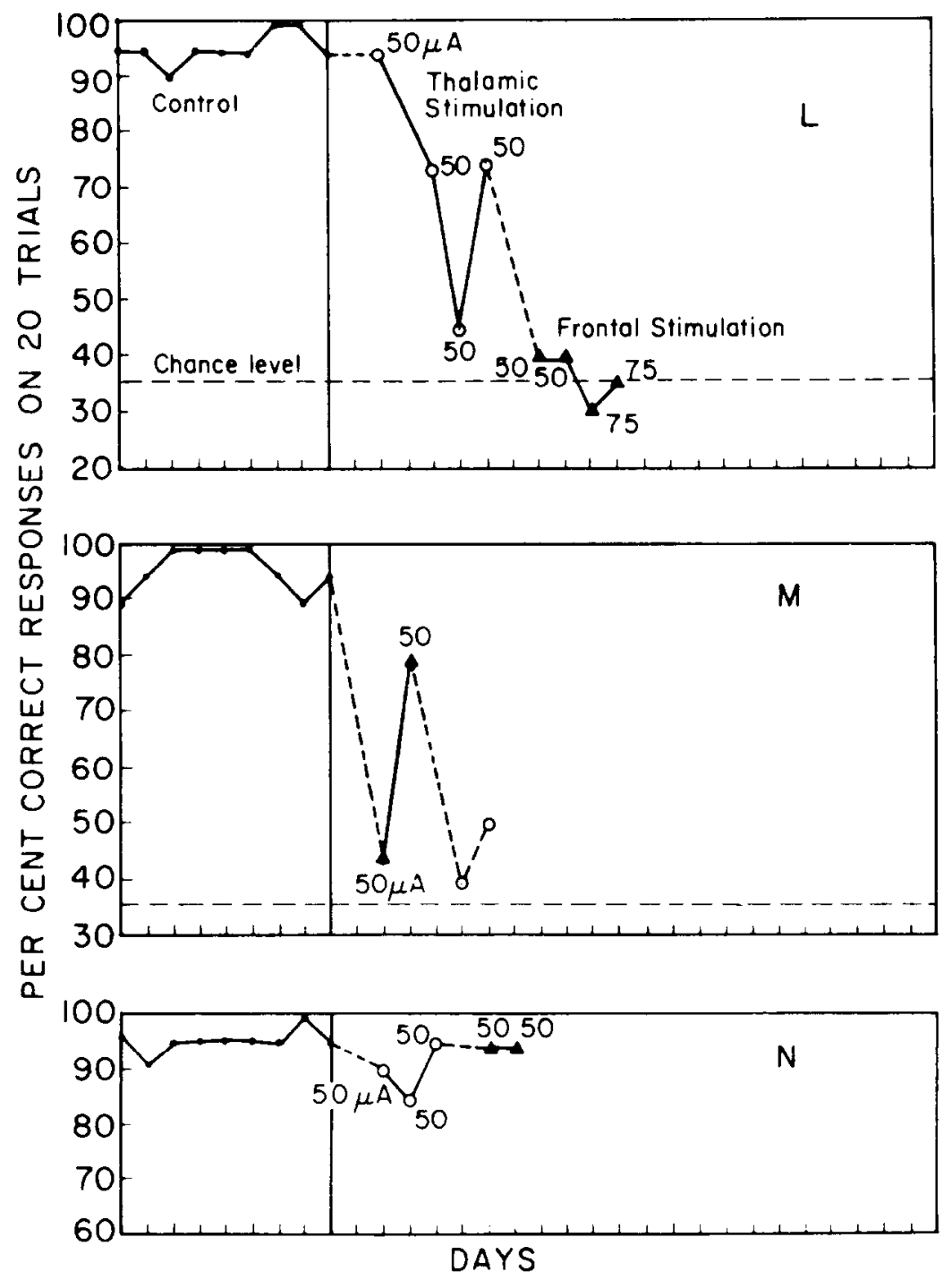

Fig. 5. Effects of intermittent stimulation throughout delayed response test, 1 sec on, 3 sec off. 
subjects tested at currents lower than those used to cause impairment when stimulation was applied during the delay period. In subjects $M$ and $L$, in which stimulation caused impairment, performance was brought to chance levels by currents below those causing motor artifacts or

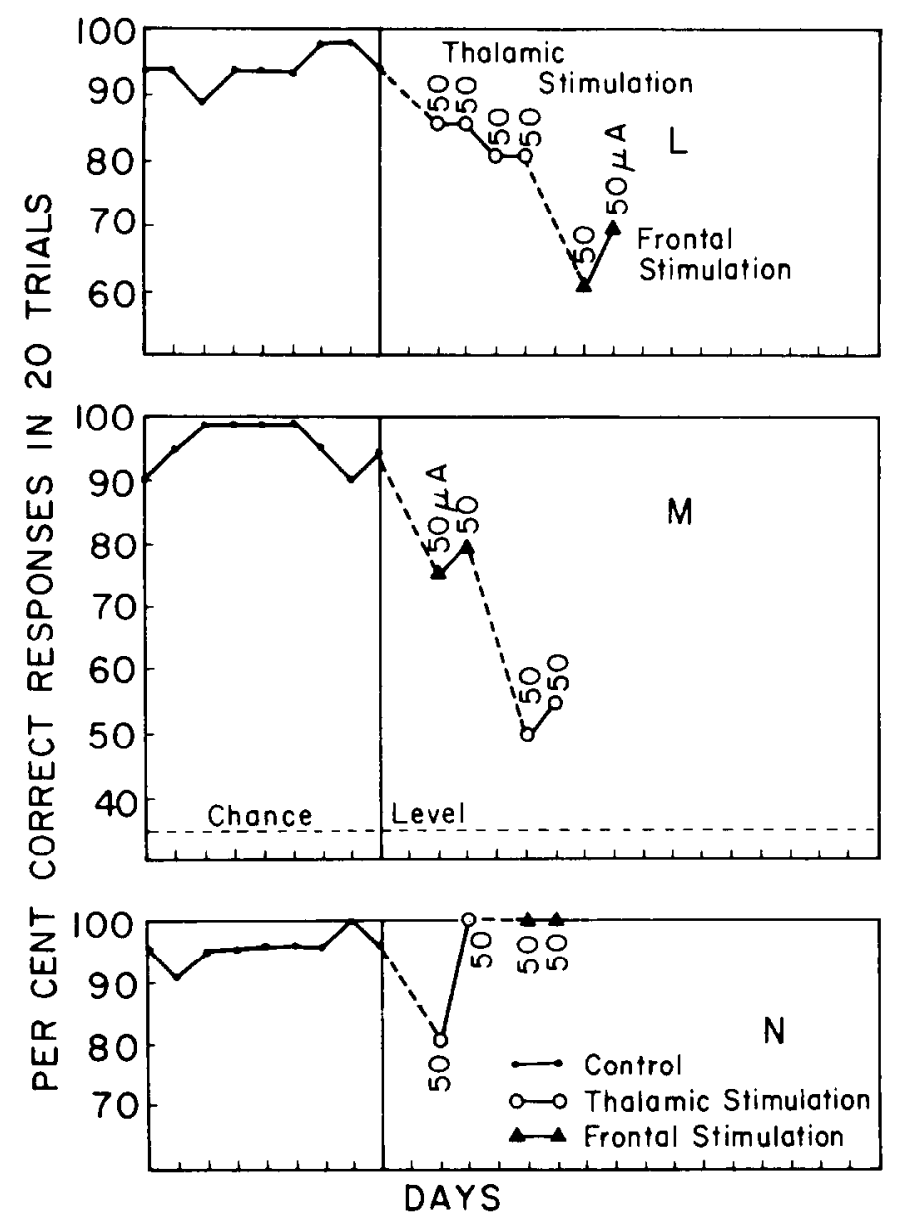

FIG. 6. Effects of continuous stimulation with $60 \mathrm{cycle} / \mathrm{sec}$ sine wave throughout delayed response test.

afterdischarges. It seemed that this was the most effective mode of stimulation (Fig. 5). Subject $N$ 's performance was not disrupted by either thalamic or frontal stimulation. Possibly stimulation at higher currents would have caused deficits, but when these were tried the subject had seizures. 
Finally, continuous stimulation with 60-cycle current of either prefrontal or thalamic points throughout the test caused less impairment than did either intermittent stimulation throughout the test or stimulation during the delay period. Surprisingly, the deficits were in the same range as those caused by stimulation during the baiting period (Fig. 6).

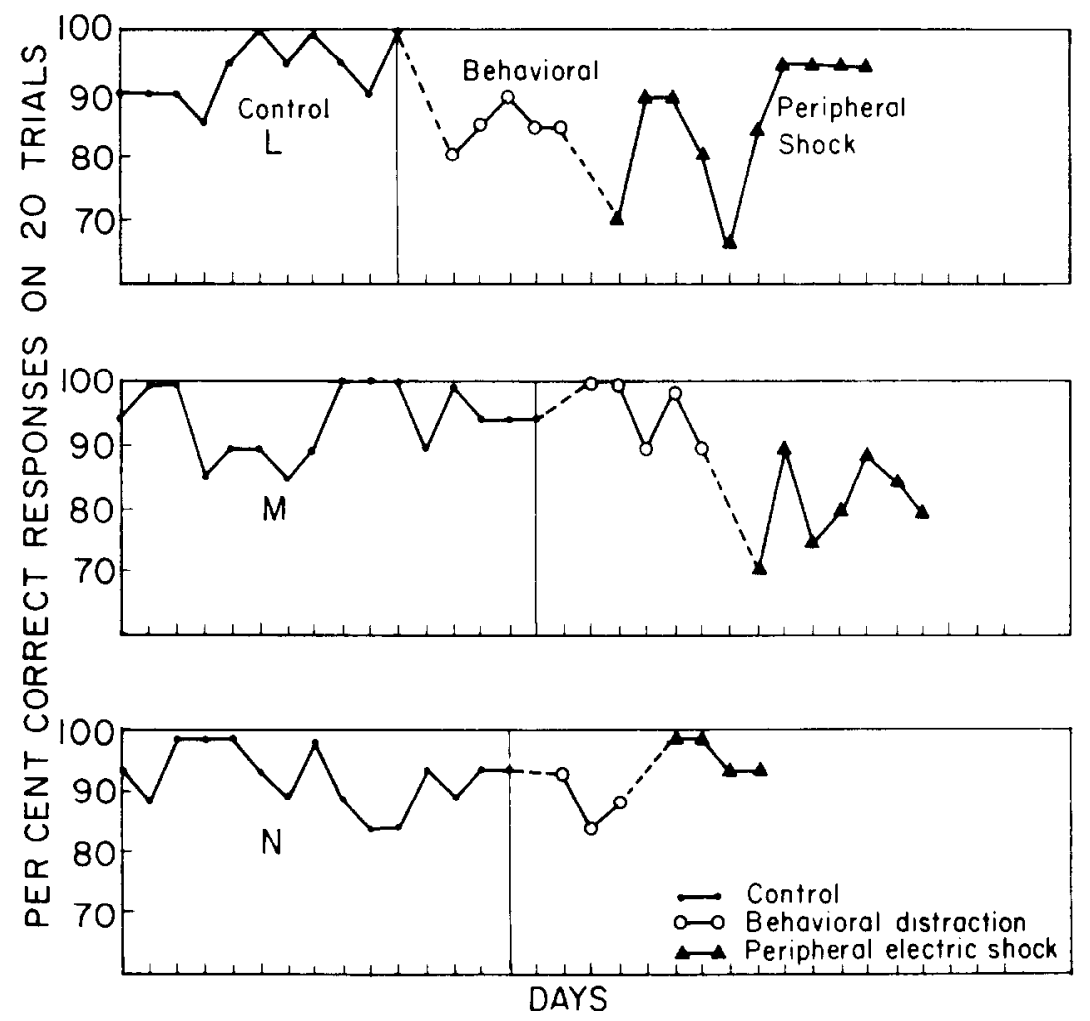

FIG. 7 . Behavioral and electrical distraction tests.

For example, under continuous thalamic stimulation, $L$ performed at the $80 \%$ level, yet discontinuous stimulation brought its performance down to the $40 \%$ level. The same difference was evidenced in the case of frontal stimulation. This difference between the efficacy of intermittent stimulation and continuous stimulation held for both $L$ and $M$. However, subject $N$ seemed very little affected by continuous stimulation. The monkey made a few more errors under thalamic stimulation, but its performance was at prestimulation levels when frontal points were excited. 
Performance during behavioral distraction tests (rattling of the primate chair, disturbing noises, etc.) was at levels achieved during control tests (Fig. 7). Subject $L$ made a few more errors regularly during such tests, but it recovered quickly, and after this even the introduction of new distractions had no particular effect. Peripheral shock through the bars of the monkey chair caused somewhat more impairment in performance, especially when these shocks were at the upper level of annoyance. Yet the

TABLE 1

Effects on Behavior of Electrical Stimulation of Individual Points in Prefrontal areas (F) and in Dorsomedial Thalamus (T). Neither Typical Self-Stmullation nor Escape Was Obvious in Any of the Subjects. Each Score Represents Number of Times Behavioral Responses Were Obtained during Stimulation of Eighteen Points in Prefrontal Areas and Eighteen Points in Thalamus. Two Scores Are Given for Each Animal, One at Threshold Current (Used in Delayed Response Test) and One Obtained at High Current

\begin{tabular}{cccccccr}
\hline & $\begin{array}{c}\text { Current } \\
(\mu \mathrm{mp})\end{array}$ & $\mathbf{F}$ & $\mathbf{T}$ & $\mathbf{F}$ & $\mathbf{T}$ & $\mathbf{F}$ & $\mathrm{T}$ \\
\hline${ }^{a}$ Moderate & 100 & 1 & 6 & 0 & 3 & 0 & 8 \\
arousal & 200 & 0 & 5 & 1 & 4 & 0 & 6 \\
${ }^{b}$ Intense & 100 & 0 & 0 & 3 & 2 & 2 & 10 \\
arousal & 200 & 2 & 2 & 5 & 7 & 1 & 8 \\
Motor & 100 & 1 & 1 & 16 & 2 & 1 & 16 \\
response & 200 & 1 & 2 & 17 & 3 & 3 & 17 \\
\hline
\end{tabular}

${ }^{a}$ Attention: The subject looks around as if trying to locate something or just looks "aware that something is going on." The animal is interested but not excited.

${ }^{b}$ Agitation: The animal is very active. There is no interest or goal-oriented behavior, but simply general activity correlated with stimulation.

${ }^{c}$ Trembling, turning left or right, general jerk with onset of stimulation, motor artifact of eyes, and coughing.

errors caused by these stimuli were not as numerous as those caused by central stimulation which did not produce any behavioral effects at all. When these peripheral shocks were attenuated, effects on delayed response were minimal and soon disappeared altogether.

For example, peripheral shock brought $M$ 's performance down to 70 to $80 \%$ correct, but frontal and thalamic stimulation which caused no afterdischarges and was scarcely noticed by the subject brought performance down to the chance level. Therefore, these data lead us to surmise that neither simple distraction nor interference by peripheral stimuli to which the animal must attend can explain the impairment caused by stimulation of prefrontal or thalamic points. 
Motivational test results of each point stimulated during the delayed response task were negative insofar as self-stimulation and escape behavior were concerned: The subjects did not learn voluntary responses to turn on or off stimulation in prefrontal or thalamic areas. On the other hand, stimulation of some individual points produced signs of slight be-

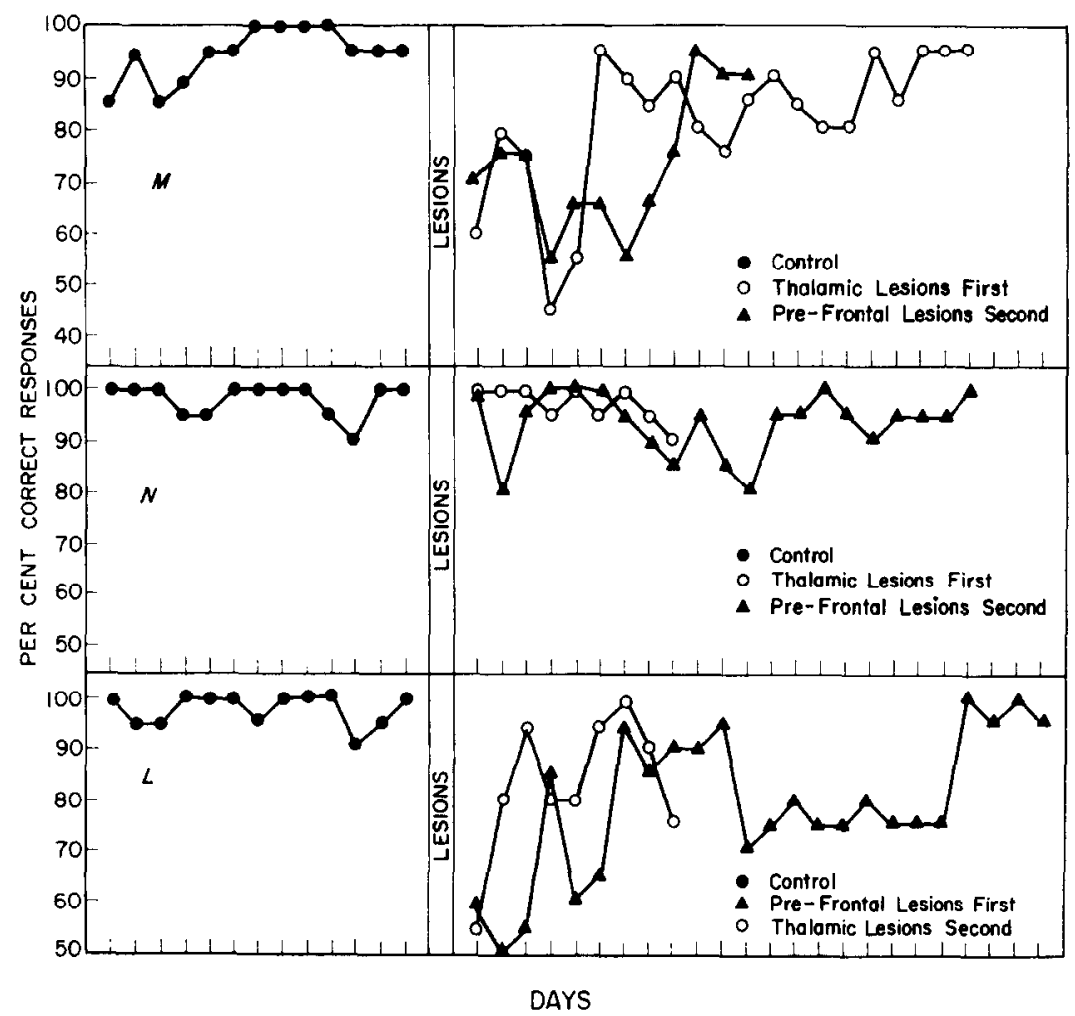

FIG. 8. Effects of electrolytic lesions in prefrontal areas and thalamus on delayed response test.

havioral activation (Table 1). While the absence of behavioral reward or punishment is not surprising in the case of frontal points, it is perhaps more tentative in the case of thalamic points. Earlier reports (1) pointed to mild reinforcement produced by stimulating some points in dorsomedial nucleus of thalamus. Subject $N$ 's performance deteriorated slightly when thalamic points were stimulated, but not when frontal points were excited, suggesting perhaps some motivational effects, although such 
effects were certainly mild. Thalamic stimulation which caused at least initially some errors in this very stable subject appeared to heighten the general activity level of this already active animal.

Prefrontal lesions initially produced longer-lasting disruptive effects than did thalamic lesions (Fig. 8). But, the order in which the lesions were received made no difference: for example, subject $L$ received frontal lesions before thalamic ones, yet its performance after receiving frontal lesions was disrupted for several weeks. However, when thalamic lesions were made subsequently, and thus $L$ had now eighteen prefrontal lesions and eighteen thalamic lesions, its performance was impaired for only a short time. By the third testing day, L's performance was $95 \%$ correct, whereas it had been $85 \%$ correct after frontal lesions, and subsequently fell to $60 \%$. Frontal lesions were made in subject $M$ after we had investigated the effects of thalamic lesions. In this case, too, frontal lesions produced longer-lasting disruptive effects than did thalamic lesions. Finally, in subject $N$, whose performance was only slightly disrupted by thalamic stimulation and not at all by frontal stimulation, thalamic lesions produced no deficits, but frontal lesions impaired its performance. Thus, electrocoagulation of prefrontal points was more effective than electrocoagulation of thalamic points in causing deficits in delayed response performance.

The interesting point evidenced by all threc subjects is that they could achieve a $90 \%$ correct performance on the delayed response test within a few weeks of receiving multiple lesions both in frontal areas and in dorsomedial nucleus of thalamus. Hence, the disrupting effects of such lesions, while quite severe, are only temporary.

\section{Discussion}

The results of these experiments substantiate the conclusion reached by Weiskrantz et al. (6) that electrical stimulation of points along the banks of the principal sulcus in the monkey can disrupt delayed response behavior of overtrained subjects, but, on the other hand, stimulation in the dorsomedial nucleus of the thalamus clearly has the same effect, as tentatively suggested by Cianci (2) and Briese and Olds (1). Though it might be argued that disruption in prefrontal areas is greater or obtains at lower intensities of stimulation, data obtained in the present studies do not lend sufficient evidence to warrant this conclusion. Electrical stimulation appears to disrupt memory processes rather than to 
inhibit performance, or reduce drive, or introduce distracting stimuli. Even when stimulated, subjects performed at the normal speed, and during no stimulation test sessions did the animals refuse to perform the twenty trials, evidencing the same eagerness to perform as shown during the training period and on alternating control test days.

With electrical stimulation, the impairment was essentially the same whether the stimulation occurred during the delay period or during the baiting period, when the stimuli lasted $0.25 \mathrm{sec}$ of every second, or throughout the test, when stimuli lasted $1 \mathrm{sec}$ out of every $4 \mathrm{sec}$, or when stimuli were continuous 60 -cycle sine waves. The four modes of applying electrical stimuli differ in their degrees of effectiveness, but it is difficult to determine whether intermittent stimulation throughout the test period was most effective because of the order of application, or because of some special added effect of stimuli which lasted $1 \mathrm{sec}$ out of every $4 \mathrm{sec}$. The surprising fact demonstrated by the four modes of stimulation is that continuous stimulation applied last in the testing sequence proved less effective than intermittent stimulation or stimulation during delay.

There is no clear-cut difference in the magnitude of impairment caused by thalamic stimulation or frontal stimulation. When applied first, stimulation in the dorsomedial nucleus of the thalamus caused errors of the same range as those caused by stimulation along banks of principal sulcus. Frontal stimulation may be slightly more effective in causing deficits; on the other hand, thalamic stimulation had some effect in all three subjects, even in subject $N$ in which prefrontal stimulation had no effect.

The data of these experiments support the notion of memory deficits caused by electrical stimulation in prefrontal areas. However, this interpretation is perhaps more open to doubt so far as stimulation in thalamic areas is concerned. It is possible that deficits which occurred during thalamic excitation were caused by the emotional properties of the points excited or by the modification of the arousal state of the subjects. Yet because of the similarity of the deficits caused by thalamic and frontal stimulation, and further because of the well-known anatomical connections between these two structures, it is likely that deficits produced by thalamic stimulation were due to some interference with the subjects' plan or strategy for performing the delayed response test.

Some question arises regarding the lack of effects of frontal stimulation on subject $N$, in which thalamic stimulation had only mild disruptive 
effects. Monkey $N$ was the only one that could be described as hyperactive, easily distracted, and most likely not to notice cues during the baiting period. Yet, this subject performed at constantly high levels whatever the mode of stimulation. Possibly the lack of effects was due to some strategy, mode of remembering, or cues subject $N$ employed to perform the delayed response task that were not employed by the other two subjects. Subjects $L$ and $M$ were always attentive and calm; even when stimulated by any of the four modes, these two subjects always gave the same general appearance of attention and interest that they had shown during training and control sessions. In the moving, hyperactive subject $N$, electrical stimulation had no effects; in the fully attentive, calm subjects, stimulation caused disruption. Hence, it is possible that the subjects in which performances were impaired by excitation were remembering with strategies different from those of the subject which showed no apparent effects from excitation. Some modes or plans of remembering could be disrupted by stimulation, others could not.

With regard to a subcortical-cortical system mediating the delayed response performance, the studies of animals with lesions, in addition to the stimulation work, implicate the dorsomedial nucleus of thalamus. Stimulation and lesions in these nuclei caused impairment of performance on the delayed response test, but these results are not too surprising in view of the projections to the frontal cortex shown by studies of retrograde degeneration (5). But while such a system subserves delayed response performance, it is not always "necessary" to the mediation of this behavior in view of the fact that recovery after lesions is possible and performance can return to pre-lesion levels. Perhaps more than one corticosubcortical system mediates delayed response-type behavior and that when the corticothalamic system is not available, some other system is available to compensate.

\section{References}

1. BRIESE, E., and J. OLDS. 1964. Reinforcing brain stimulation and memory in monkeys. Exptl. Neurol. 10: 493-508.

2. Cianci, S. N. 1965. Effects of cortical and subcortical stimulation on delayed response in monkeys. Exptl. Neurol. 11: 104-114.

3. Rosvold, H. E., and J. M. R. Delgado. 1956. The effect on delayed alternation test performance of stimulating or destroying electrically structures within the frontal lobes of the monkey's brain. J. Comp. Physiol. Psychol. 49: 365372 . 
4. Sтамм, J. S. 1961. Electrical stimulation of frontal cortex in monkeys during learning of an alternation task. J. Neurophysiol. 34: 414-426.

5. Warren, J. M., and K. Akert. 1964. "The Frontal Granular Cortex and Behavior." McGraw-Hill, New York.

6. Weiskrantz, L., L. J. Mihailovic, and D. G. Gross. 1962. Effects of stimulation of frontal cortex and hippocampus on behavior in the monkey. Brain 85: 487-504. 